\title{
Use of Simple Mobile Technology to Create an Electronic Vaccination Registry in Kenya
}

\author{
Samuel Juma ${ }^{1,3 *}$, Collins Tabu ${ }^{2}$, Zainab Gura ${ }^{3}$, Samuel Waweru ${ }^{4}$, Ian Njeru ${ }^{3}$ \\ ${ }^{1}$ School of Public Health, Texila American University, Guyana \\ ${ }^{2}$ National Vaccines and Immunization Program, Ministry of Health, Nairobi, Kenya \\ ${ }^{3}$ Field Epidemiology Society of Kenya, Nairobi, Kenya \\ ${ }^{4}$ Department of Health Information Systems, County Health Services, Nyandarua, Kenya
}

\begin{abstract}
Vaccination is one of the high-impact public health interventions against the spread of disease. Over time, developed countries have been able to reduce the burden of disease through improving access to vaccination and achieving high vaccine coverage. In low-income countries, the situation is different as most countries still report low coverages of less than 90\%, which is the global target recommended by the World Health Organization. The main reasons for this low coverage include poor access to vaccination, stock-outs, and poor documentation and targeting for vaccination services. To address these problems, we developed an electronic vaccine registry using Unstructured Supplementary Service Data (USSD) technology that registered births, vaccines administered and sent short message reminders to mothers about their clinic dates. The study was conducted in Nyandarua County, Kenya, between June 2018 to March 2019. To participate in the study, mothers had to reside within the jurisdiction of the study site. Mothers who moved into the study site also had their children registered and previous vaccines updated. A total of 4,823 births and 20,515 vaccines administered were captured into the system. The system sent 12,554 short message reminders to mothers; 3 days before the due day and on the due day. Additionally, it generated a birth register, vaccination register, defaulter list, dropout rate report, vaccine coverage, and timeliness reports. The intervention improved vaccination coverage and timeliness of vaccination by up to $8.7 \%$.
\end{abstract}

Keywords: Coverage, Immunization, Registry, Vaccination.

\section{Introduction}

Vaccines have been known to be one of the best public health interventions with the highest impact of reducing diseases, disability, and deaths [1]. Vaccines also result in increased economic productivity and net economic benefits by saving billions of dollars in illness costs worldwide [1]. These benefits have largely been realized in many of the developed countries like the USA and European countries, where vaccination coverage is more than $90 \%$. However, many low- and middle-income countries, especially in Sub-Saharan Africa, are yet to achieve a vaccination coverage of $80 \%$ for the fully immunized child [1]. On average, one in 5 children in Africa is unvaccinated [2].

In 2016, the World Health Organization estimated that about $85 \%$ of the world infants were vaccinated against Diphtheria, Tetanus, and Pertussis (DPT), Hepatitis B, Measles and Polio thereby preventing approximately 2-3 million deaths, although an additional 1.5 million deaths could be prevented if vaccination coverage improves [3]. By 2019, the coverages had dropped slightly to $81.6 \%$, as did global coverage of the first-dose measlescontaining vaccine, MCV1, to $83.6 \%$ [4]. However, the vaccination coverages varied by countries with Kenya being estimated at $89 \%$ 
for DTP3 and $75 \%$ for Measles Containing Vaccine 1(MCV1) [5].

There are many reasons for low vaccination coverage, and this varies from country to country. The reasons could be related to poor access to services (e.g., distance to the health facility, financial challenges), poor communication of information by health workers (e.g., date of next visit), low literacy levels of parents, and non-prioritization of vaccination by parents due to socio-cultural issues such as religious beliefs and attitudes that are against vaccination. Other reasons cited include home deliveries, poor monitoring by health care workers, poor social-economic status [6-10]. Though all the above factors can be a cause of low vaccination coverage, it has been noted that routine immunization coverage in many low- and middle-income countries has stagnated in the last several years despite various interventions aimed at addressing the above challenges [3].

One important reason that could explain this stagnation is the quality of data that is used to estimate the coverage. Most of the time, the vaccination coverage is estimated based on administrative data, which uses an estimated denominator for the number of children. Since the denominator is an estimate that is based on a census that may have been conducted several years back, the administrative vaccine coverage may therefore be either overestimated or underestimated when compared with demographic survey data that is deemed more accurate.

One way to overcome the denominator challenge and therefore provide better vaccination coverage is to create an electronic vaccination registry. An electronic vaccination registry (EVR), also known as an immunization information system (IIS), is defined as a 'population-based, computerized information system that collects and consolidate vaccination data from multiple healthcare providers within a geographic area' [11]. An EVR can greatly help to increase vaccine coverage by providing the denominator of all children living in a geographical area. It also helps to consolidate vaccination records of children from multiple health providers and therefore provides more accurate data. An EVR also helps to increase vaccination coverage by providing SMS reminders. It also helps to monitor vaccination coverage on an individual level and therefore helps to improve the timeliness of vaccination as well as coverage [12].

Due to financial and technological challenges, it has not been possible to create electronic vaccination registries in many countries and especially in Africa. The electronic vaccine registries routinely created are web-based and require comprehensive information communication and technology equipment like laptops, tablets/smartphones, and internet accessibility to use. This study therefore aimed at creating a simple and costeffective electronic vaccination registry that uses simple technology (Unstructured Supplementary Service Data or USSD technology) that can easily be scaled up to many geographical areas to improve timeliness and completeness of vaccinations. The electronic vaccine registry created using USSD technology only requires a mobile phone network and an ordinary phone, and this makes it cheaper and more accessible to users.

The main objective of the study was to determine the feasibility of creating an electronic vaccination registry using USSD technology to increase the timeliness and completeness of vaccination for children under 5 years.

The specific objectives were:

1. To digitize the birth register in near realtime using SMS (USSD) technology to provide vaccination denominator for automated SMS reminders.

2. To digitize the vaccination, register in near real-time using SMS(USSD) technology.

3. To determine the impact of using a vaccination registry on the timeliness of vaccinations. 
4. To determine the impact of using a vaccination registry on individual and population vaccination coverage.

\section{Materials and Methods}

\section{Study Design}

The study was a prospective cohort where all children born in the study area during the study period were recruited into the study. Every child in the birth cohort was followed up for a minimum of 4 months to monitor how they received their early childhood vaccinations. The outcomes (timeliness and completeness of vaccination before and after intervention) were compared with a control cohort randomly selected from another sub-county in the study county.

User requirements were gathered from health workers who administer vaccines. This was then followed by the development of system requirements specifications and designing of the system. Births that occurred at home were recorded by local administrators (chiefs and sub-chiefs) in manual registers as usual. These births were then recorded (within 2 days) in the electronic system by public health technicians (PHTs) or public health officers (PHOs) who liaised with local administrators (birth notification number was used as the unique identifier). Health workers at health facilities recorded births and vaccinations in their manual registers as usual and then recorded the data in the electronic system.

This intervention focused on digitizing the birth register (also known as Form B1) and the permanent immunization register (also known as MOH 510). Form B1 is usually filled by the health workers for children born at health facilities and the local administrators (chief or sub-chiefs) for children born at home. These forms are then usually submitted to the subcounty civil registration departments when the books fill up, which most of the time is many months after the critical immunization period, which is the first 14 weeks of a child after birth. The intervention also focused on digitizing the permanent register, which is normally based at the vaccination clinics and captures the child's vaccination history from birth to 18 months.

Once birth notification forms were issued, the data was digitized within 24 hours so that it is available in the system when the child goes for the birth vaccines. Data in the permanent immunization register was digitized at least twice a week. Digitizing Form B1 within 24 hours after it is issued provided a near real-time birth denominator that is currently missing at health facilities for accurate calculation of population-based vaccine coverage.

The system used the Form B1 serial number as the unique ID for the child, and every effort was made to ensure that adequate Form B1s with unique serial numbers are available at the hospitals and with the local administrators in the villages. The unique serial numbers were used to identify and integrate the vaccination record of a child irrespective of which health facility the child was vaccinated.

A basic phone was used to register births and record vaccines given to a child. Local administrators (chief or sub-chief) recorded home births while a health worker registered children born at a health facility. The phone at the health facility was also be used to record vaccines administered at the health facility. Data collected from the mobile phones were then relayed to a central server located at Ministry of Health. Health workers either at the sub county level or health facility level were able to access the server through a computer to generate reports. The server was linked to an SMS gateway that was used to send automated SMS reminders to parents. 


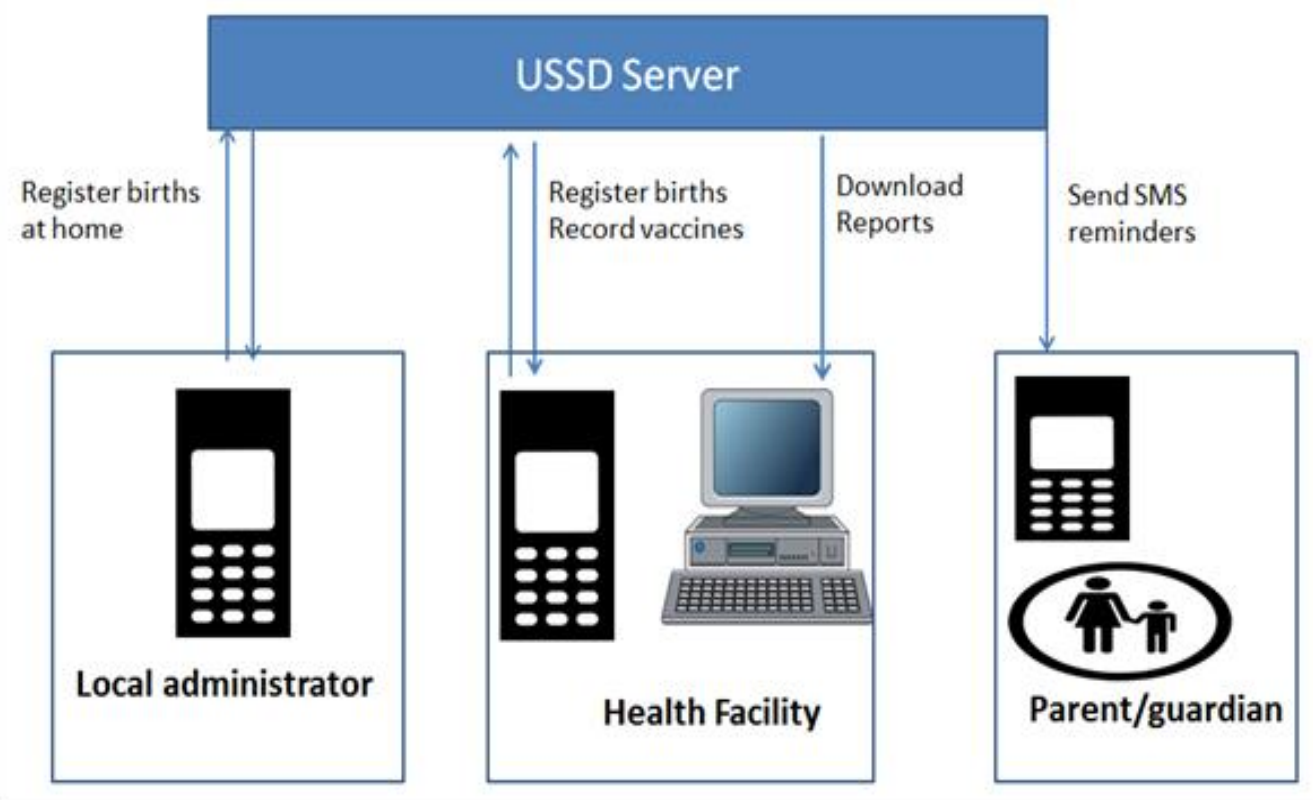

Figure 1. Operational framework; system architecture of the created system (Chiefs and assistant chiefs)

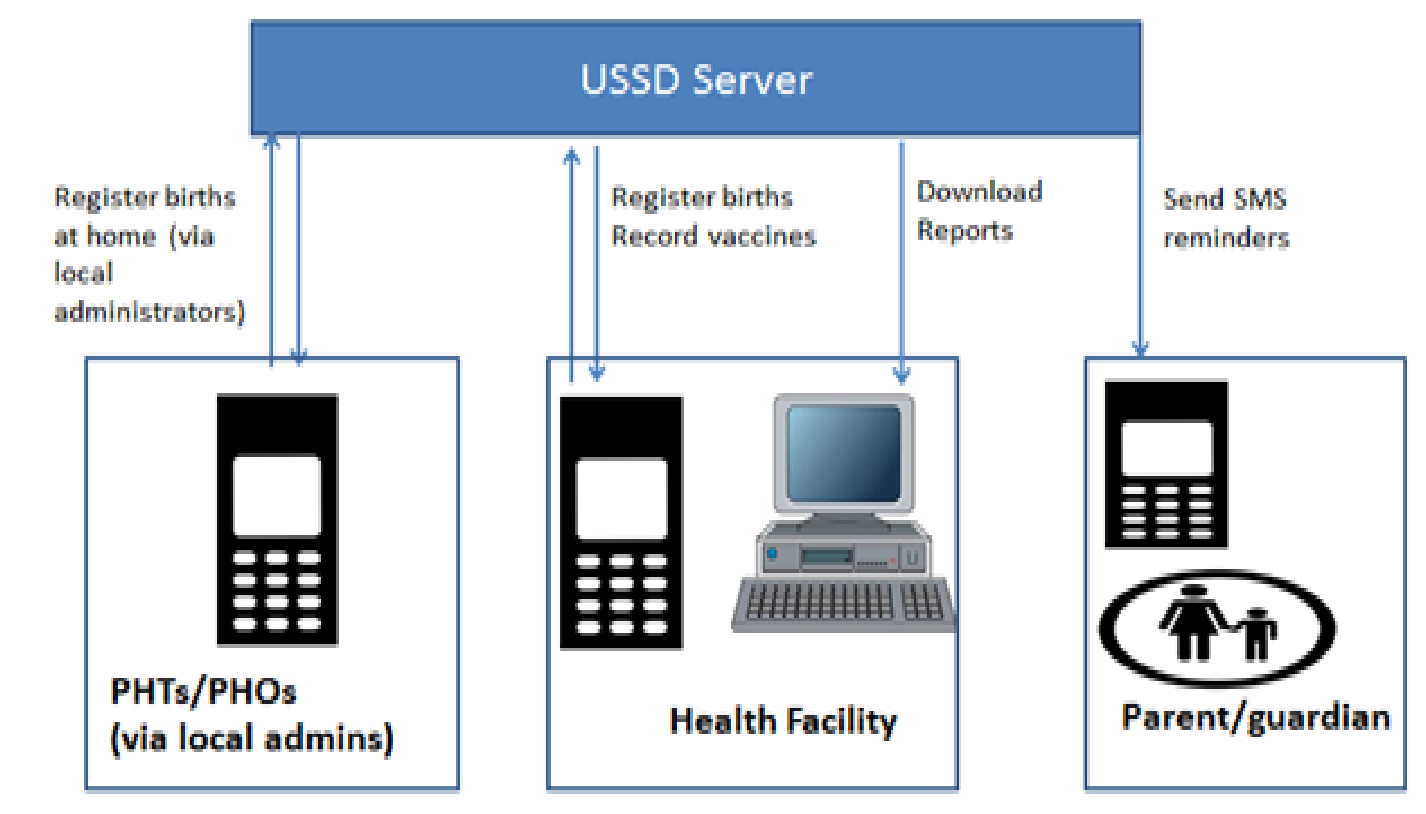

Figure 2. Operational framework; system architecture of the ChanjoTrack (PHTs)

\section{Unstructured Supplementary Service Data platform}

This is the front-end user platform that was developed and was accessed through a mobile phone using the shortcode $* 384 * 80$ \# that was operated through one of the mobile network operators in Kenya by dialing the shortcode, and one could perform several tasks such as register births, record vaccines, record deaths, transfer out, query birth registration status and query vaccination status.

\section{Reporting Platform}

We also developed a website where detailed reports could be accessed and downloaded by users. Through this website users could access various reports including birth register, immunization register, defaulter list, 
vaccination reports, timeliness reports, dropout reports, and vaccination coverage.

\section{SMS Reminders to Parents}

The ChanjoTrack platform sent automated and customized reminders to the mothers. Two SMS reminders were sent: the first one was sent 3 days to the due date, and the second one was sent on the due date.

\section{Study Site}

The study was conducted in Ndaragwa subcounty in Nyandarua County, which is in Central Kenya. Nyandarua County has 5 sub counties as follows: Kinangop, Kipipiri, Ndaragwa, Oljororok, and Ol kalou. According to the DHIS2 data, in 2017, the 5 sub-counties had estimated fully immunized child coverage as follows: Kinangop $80 \%$; Kipipiri $60 \%$, Ndaragwa 58\%, Oljororok 68\%, and Ol Kalou $78 \%$. As Ndaragwa had the lowest coverage, the sub-county was selected, and all health facilities in the sub-county participated in the study. The county was selected based on 2 parameters:
1. Relatively high access to mobile phone use among the residents and therefore good opportunity to test the electronic vaccination registry which relied on phone availability to send messages to parents/guardians. Nyandarua County had $60 \%$ access to phone use in the 2009 population census compared to $51 \%$ nationally (KNBS 2016).

2. Vaccine coverage in Ndaragwa was lower than the national target of $90 \%$ by administrative data. Administrative vaccine coverage for Nyandarua in 2017 was approximately $73 \%$ for Penta 1 (1st dose of Diphtheria, Tetanus, Pertussis, Hepatitis B, and Haemophilus influenza B vaccine), $69 \%$ for Penta3 and $71 \%$ for the fully immunized child (DHIS2 2018). This coverage is markedly lower than the Kenya Demographic Health Survey findings of 2014, which showed Nyandarua County had vaccine coverage of $100 \%$ for Penta 1 , $91 \%$ for Penta2, and $81 \%$ for fully immunized [13].

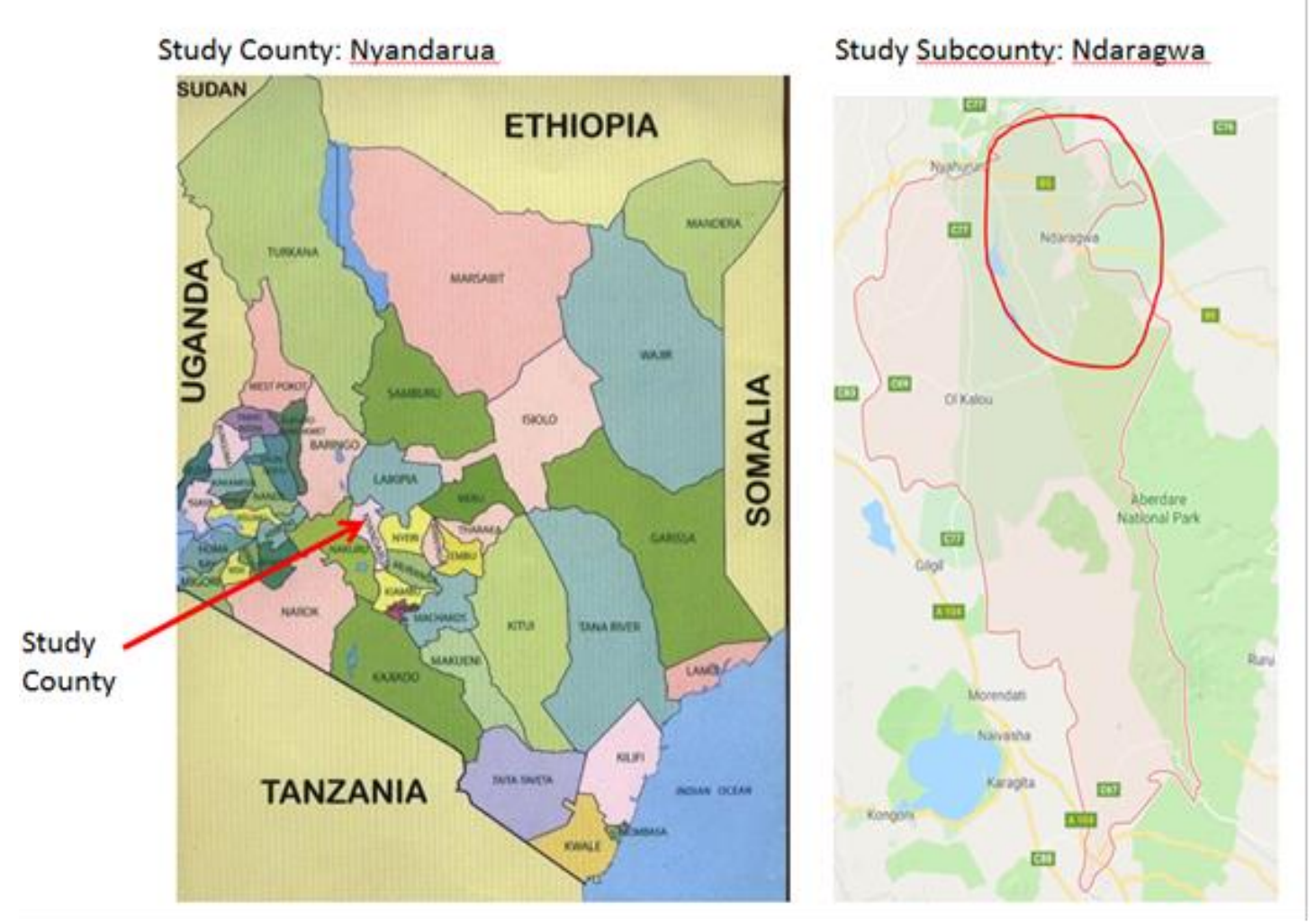

Figure 3. Map of Kenya showing the study site in Nyandarua County, Ndaragwa 


\section{Study Population (Inclusion and Exclusion Criteria)}

The study population included all children that were born in the study area during the study period (June 2018 to April 2019).

\section{Exclusion Criteria}

1. Children whose parents/guardians declined to take part in the study.

2. Children who were born before the study and were therefore continuing with vaccination services. They were be excluded as it will was difficult to get their birth data, especially the birth notification ID/serial number, which was required by the electronic vaccination registry.

\section{Sample Size Calculation}

The study assumptions were as follows:

1. This was a prospective study (unmatched cohort), and all children born in the study area were be enrolled.

2. The outcome of interest for sample size calculation is the proportion (\%) of children vaccinated on time for the oral polio birth dose (OPV0).

3. Study power of $80 \%$.

4. 2-sided confidence level of $95 \%$.

Using the above assumptions, the minimum sample size was estimated to be 151 for the study group and 151 for the control group.

\section{Data Collection}

Data was collected through extraction from the USSD created vaccination registry for the intervention group and from the traditional government Health Information Management System (HIMS) for the control arm, using a data extraction tool.

A survey was also conducted among the study participants from the immunizing facilities to collect data on the acceptability, functionality, and adaptability of the USSD created electronic vaccination registry.

\section{Training of Research Assistants and Piloting}

The electronic data capture tool was tested extensively by the research team before it was taken to the field for use by end-users (health workers and local administrators). The endusers of the system were then trained for one day. During the training, the end-users used dummy data to test the system until they were fully conversant to use the system.

\section{Management of Data Quality during Field Work}

Health workers and local administrators who would be using the electronic system were trained well before they started using the system. They also used dummy data for a few days using a test server until they were comfortable to migrate to the live system. Field supervisors drawn from the Sub County health department were also recruited to assist the endusers to ensure that the data captured electronically matches what is in the manual registers.

\section{Data Analysis}

The following reports were automated from the electronic vaccine registry:

1. Birth register of all children born in the study area.

2. Vaccination registers of all children who received vaccines.

3. Number of children vaccinated per antigen(vaccine) per given time period and geographical location.

4. List of children defaulting on vaccination.

5. Proportion of children vaccinated on time by antigen(vaccine).

6. Proportion of children vaccinated by antigen(vaccine) and time period.

7. Dropout rates for certain vaccines.

i. Births - pental dropout rate.

ii. Penta1- Penta3 dropout rate. 
iii. Penta1-MeaslesRubella1 dropout rate.

iv. Measles Rubella 1 - Measles Rubella 2 dropout rate.

Data downloaded from the electronic database were analysed for two main outcomes; timeliness of vaccination (Proportion of children who receive vaccination on time per antigen) and vaccination coverage (Proportion of children who are vaccinated by antigen). The data was cleaned and analysed using Epi Info (CDC) version 7.1.3.10. Descriptive analysis was done as well as inferential statistics through regression analysis to determine relationships between variables. Statistical significance was assessed at $\mathrm{p}<0.05$.

\section{Societal and Scientific Relevance}

Electronic vaccination registries are responsible for improving performance in vaccination coverage. Use of cheaper and easier to use and accessible technology would create even more benefits to the vaccination programming. This study has explored this new technology and made appropriate recommendations to policy makers.

\section{Ethical Considerations}

The ethical clearance was granted by Africa Medical Research Foundation (AMREF) Ethics and Review Committee (Approval P454/2018). Nyandarua county health department granted permission to conduct the study in the study health facilities. Informed consent was given by the respondents after explaining the purpose and importance of the study. Confidentiality was maintained throughout the study by ensuring that respondents' names or identifications did not appear in the questionnaires and that the data collected and captured was stored in a safe passwordprotected database. The respondents' right to correct information was always ensured, and their participation in the study was voluntary, and they were free to withdraw at any point in the study if they so wished.

\section{Results/Findings}

\section{Creation of the Electronic Vaccine Registry (ChanjoTrack)}

We successfully used USSD technology to develop an integrated electronic system that registered births, recorded vaccines, and sent automated SMS reminders to parents.

Figure 4 below is a screenshot of the USSD platform and a dummy sample (not the real name of a person in the system) of an SMS reminder.
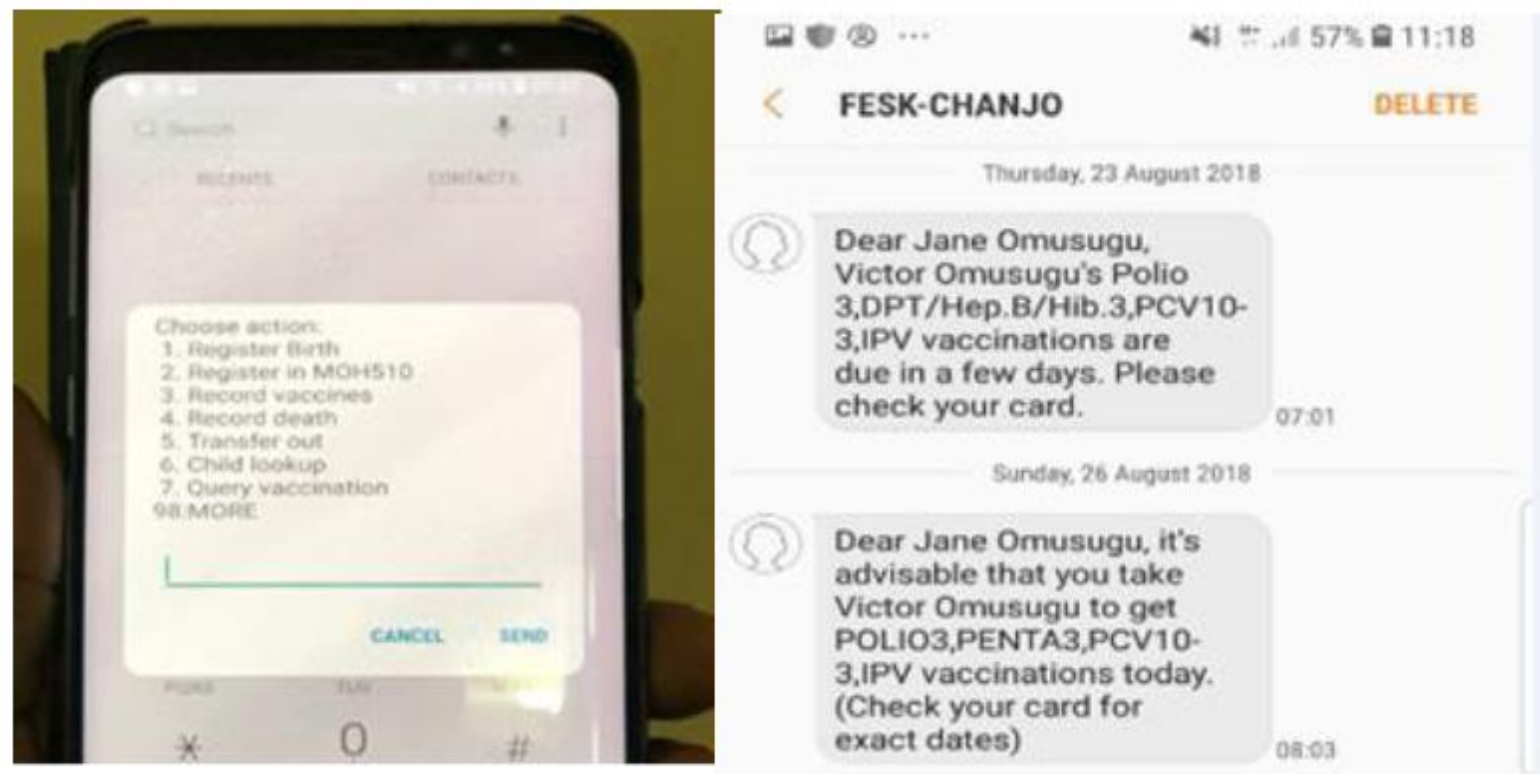

Figure 4. Map of Kenya showing the Study Site in Nyandarua County, Ndaragwa 
Data Generated

\section{Births Recorded}

A total of 4,822 children/births were registered in the platform prospectively and retrospectively from 1st June 2018 to 31 st March 2019. The births were distributed as shown in Figure 5.

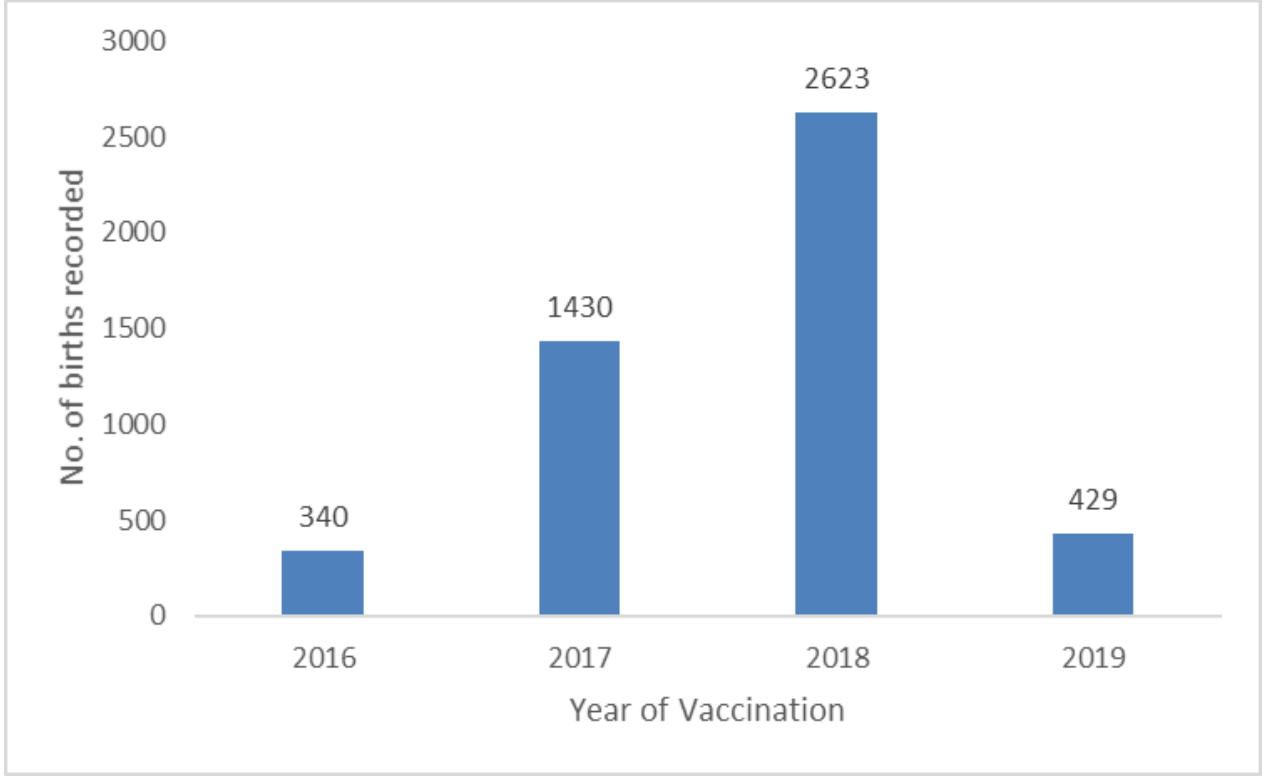

Figure 5. Number of Births Recorded into the System during the Study Period

\section{Vaccines Recorded}

The platform was successfully used to record vaccines for all children whenever they were vaccinated. For example, between 1st June 2018 and 31st March 2019, the following selected vaccines were recorded in the system.

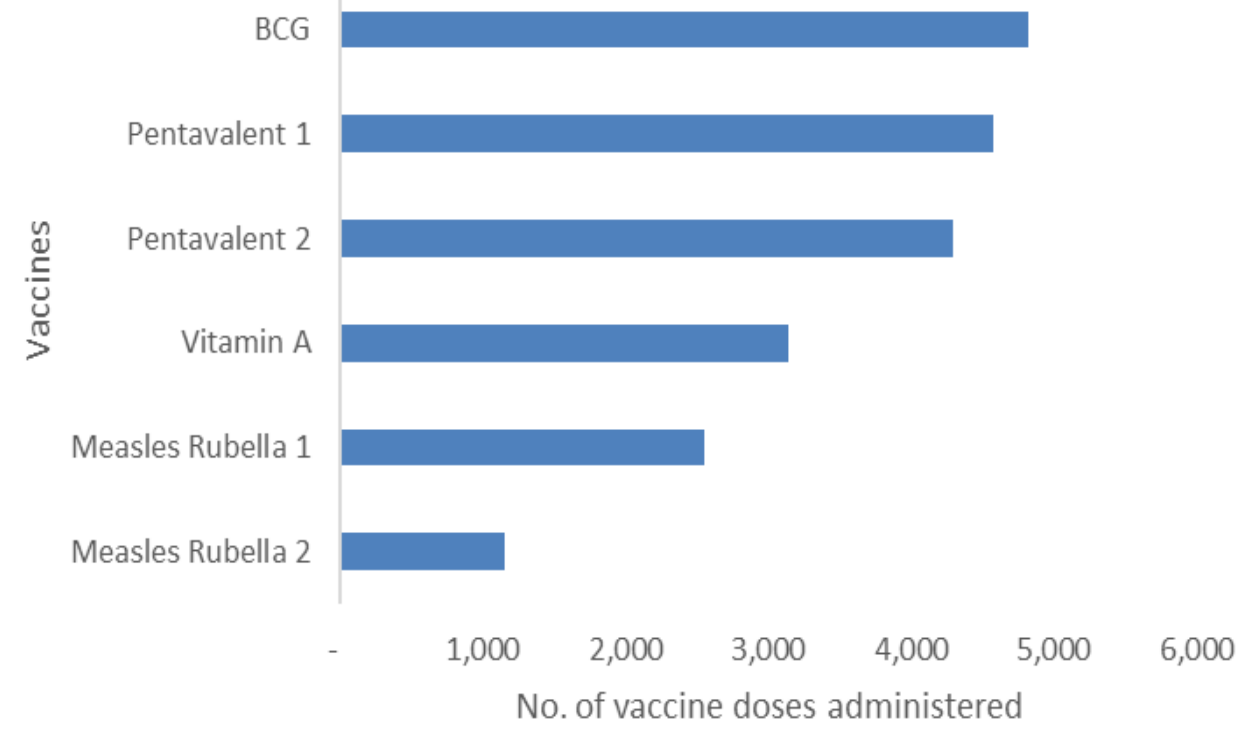

Figure 6. Number of Vaccines Administered during the Study Period 


\section{SMS Reminders}

A total of 12,554 SMS reminders were sent to parents between 1st June and 31st March 2019 as follows:

The number of SMS reminders sent 3 days before the due date was 1,464 for vaccines due at 6 weeks, 1,628 for vaccines due at 10 weeks, 1,653 for vaccines due at 14 weeks, 880 for Vitamin A due at 6months, and 764 for Measles-Rubella 1 due at 9 months.

The number of SMS reminders sent on due day was 1,412 for vaccines due at 6 weeks, 1,555 for vaccines due at 10 weeks, 1,609 for vaccines due at 14 weeks, 806 for Vitamin A due at 6 months and 784 for Measles-Rubella due at 1 at 9 months.

\section{Reports Generated}

The system was able to generate the following reports: birth register, immunization register, defaulter list, dropout report, number of children vaccinated per vaccine, vaccination coverage, timeliness of vaccination, and list of SMS reminders sent by the system.

\section{Cost of the System}

It cost $\$ 45$ to buy a phone for each of the 18 health facilities. The public health officers/technicians who assisted to register home births used their own phones. To maintain the USSD system, we paid a monthly fee of $\$ 58$. There was also a charge of $\$ 0.015$ per user per session to use the USSD system, which translated to \$3-4 per month per health facility on average.

\section{Timeliness of Vaccination}

Table 1 below shows the timeliness of vaccination before and after the project for selected vaccines given at birth, 6 weeks, 10 weeks, and 14 weeks. From the findings, timeliness was found to be relatively high in the intervention group even before the project, even though significant improvements have been reported on the timeliness for some antigens.

\section{Vaccination Coverage}

The system captured all individual vaccinations given to a child and hence improved documentation. Due to the improved documentation, the vaccination coverage for most antigens was much higher than what was reported in District Health Information System (DHIS2). For example, out of the 2623 children/births captured in our system for 2018 , $99.8 \%, 97.7 \%$ and $92.8 \%$ had received $\mathrm{BCG}$, DPT / HepB / Hib.1 and DPT / HepB / Hib.2 vaccines respectively. However, DHIS2 reported coverage of $58 \%, 84 \%$, and $83 \%$, respectively, for the same vaccines. Our system seemed to give better coverage that is close to the last official Kenya Demographic Health Survey (2014), which showed coverage of $100 \%, 100 \%$, and $98 \%$ respectively for $\mathrm{BCG}$, DPT / HepB / Hib.1 and DPT / HepB / Hib.2 for Nyandarua County where we did the study (Figure 7 below). 


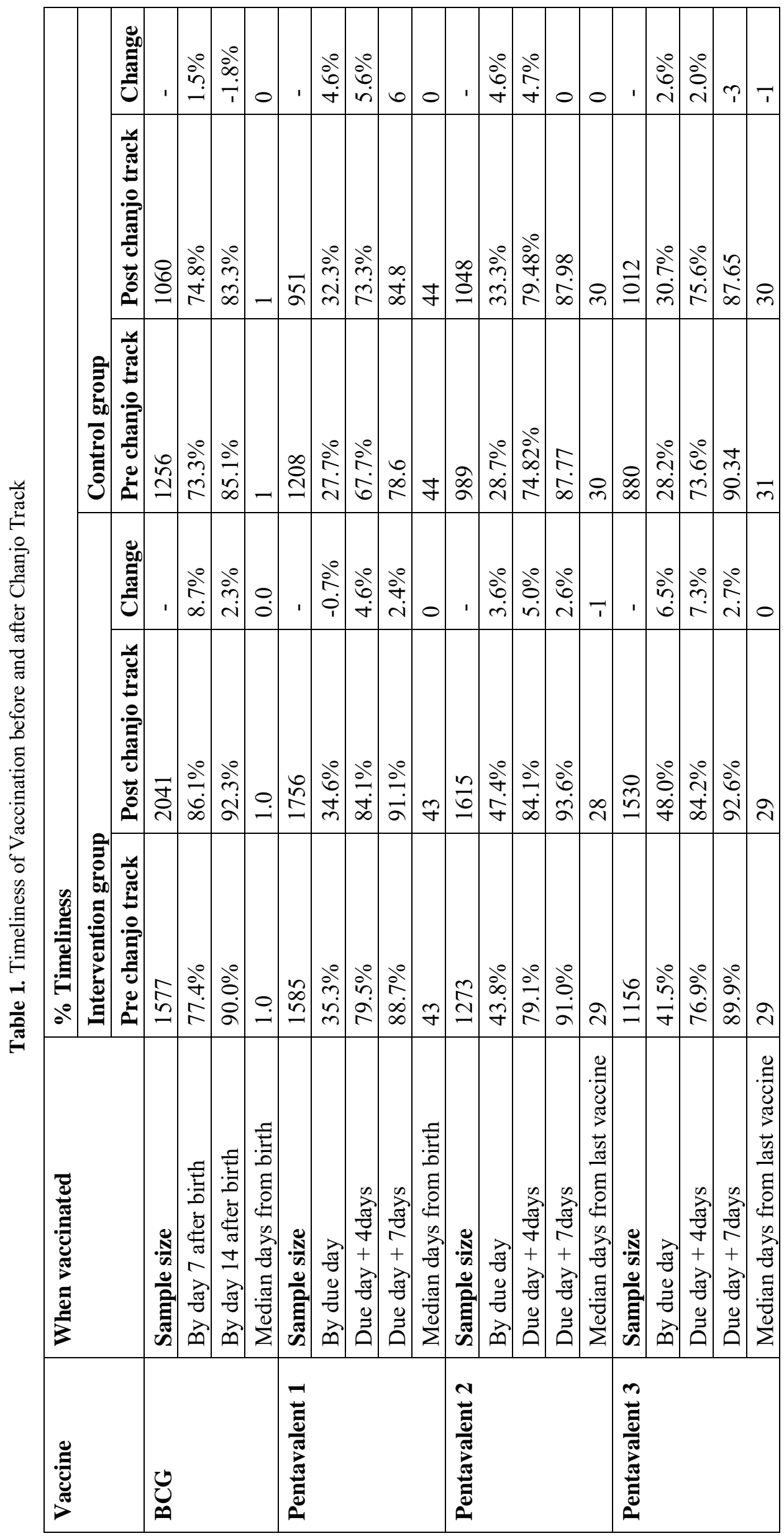




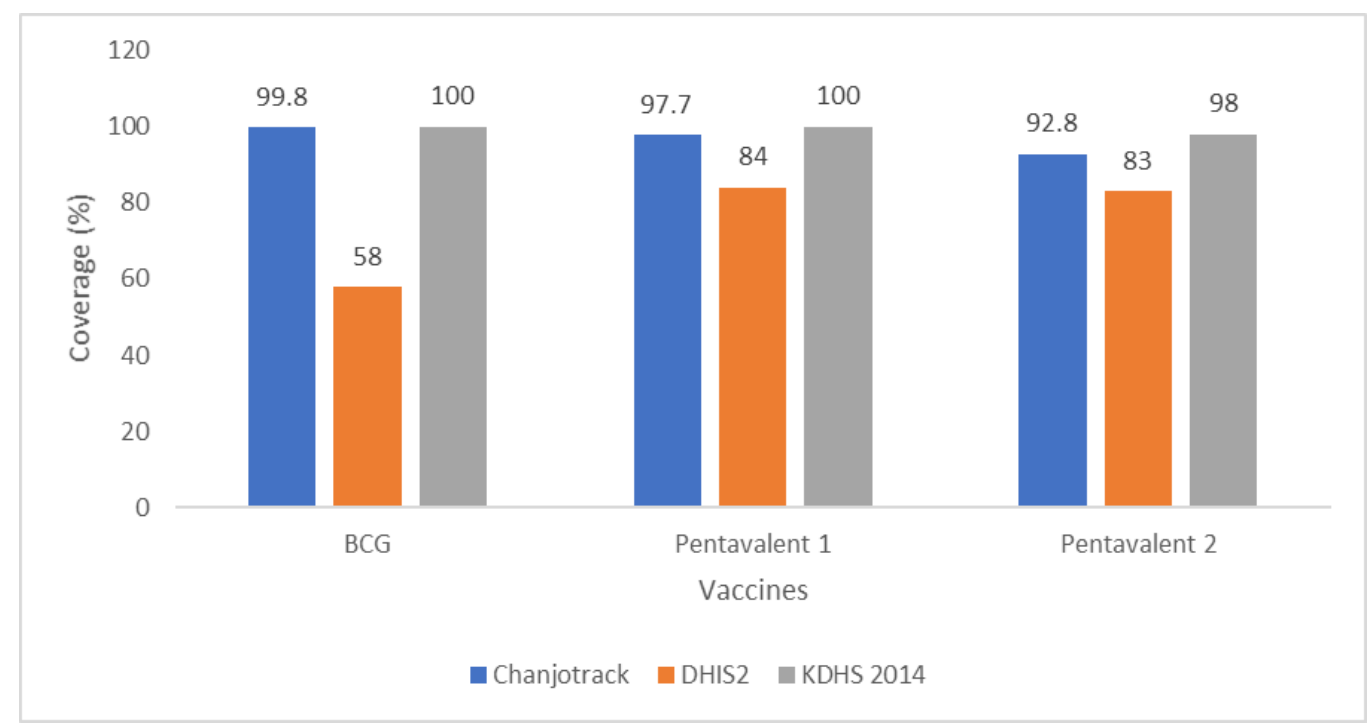

Figure 7. Vaccination coverages captured in the ChanjoTrack viz a viz KDHS and DHIS2

\section{Discussion}

The ChanjoTrack system demonstrated that a USSD system can be used successfully to create an electronic vaccine registry that is capable of registering births, recording vaccines administered, tracking vaccine defaulters, sending SMS reminders on vaccination dates, and helping establish a real-time denominator for the calculation of vaccine coverages. There is not adequate published documentation on the use of this technique, but the results indicate that it is an approach that should be explored more. This notwithstanding, lessons learned from the implementation of electronic vaccine registries summarize the composition of an ideal electronic immunization system as comprising; (i) enrollment at birth; (ii) a unique and unequivocal identifier (ID); (iii) vaccination provider, vaccine dose, and date; (iv) mechanisms for aggregating data at different geographical levels; and (v) automated individualized follow-up of vaccination schedule $[12,14]$. The ChanjoTrack met all these criteria.

Regarding improvement on vaccine coverage, ChanjoTrack gave a more accurate vaccination coverage as it was based on the actual children in the system (denominator was available). As shown in Figure 7, the coverage by ChanjoTrack was much closer to KDHS 2014 (survey data) as compared to DHIS2(administrative data). Coverage for BCG in DHIS2 was low because BCG was captured mostly where children were born e.g., a referral hospital in another county.

A study conducted to establish whether digital tools can be used to improve immunization programs lists electronic vaccine registries as one of the digital tools that improves the performance of immunization programs [15]. This is consistent with our finding on the ChanjoTrack improving immunization coverage. Another study in Vietnam also reports a significant increase in vaccine coverage a year after the introduction of the electronic vaccine registry [16].

In addition, the use of the reminder feature and notification to mothers on the clinic dates also contributed to the improved vaccine coverage. A related study conducted on utilizing health information technology to improve vaccine communication and coverage reported a similar finding. It indicated that parents reported that phone message reminders attract their attention in a different way and that they also reach the intended participant as opposed to a letter, which anyone in the household might open, or an automated telephone reminder, which will play for whom 
ever answers the phone or picks up the answering machine message. The parents also reported that messages also remain on a person's cellular phone, which is often with them, allowing potentially important reference information to be easily accessible [17]. The contribution of text message reminders to improved vaccine coverage has also been reported [18]. This is in concurrence with the findings of our study.

Timeliness in vaccination is critical in ensuring that children acquire and maintain immunity to vaccine-preventable diseases. Ideally, timeliness refers to whether a child receives a vaccine within a specific timeframe, where they can receive a dose early, on time, or late [19]. The study also reports similar findings on the ability of electronic vaccine registries to improve timeliness as they capture real-time data. In this study, the ChanjoTrack was able to improve timeliness for the various antigens administered. Compared with control facilities, the change in timeliness was slightly higher in the intervention sub-county compared to control facilities, e.g., timeliness of $\mathrm{BCG}$ vaccination (at day 7 after birth) improved by $8.7 \%$ in the intervention group compared to $1.5 \%$ in the control group. Timeliness for DPT / HepB / Hib. 2 (at 7 day after due date) increased by $2.6 \%$ in the intervention group against $0 \%$ for the control group. Children also came earlier for vaccination by 1 day compared to the pre-intervention period. Timeliness for DPT / HepB / Hib.3 (at 7 day after due date) increased by $2.7 \%$ in the intervention group against $-3 \%$ for the control group. However, Timeliness for DPT / HepB / Hib.1 (at 7 day after due date) improved better in the control group at $6 \%$ compared to $2.4 \%$ in the intervention group. As also noted in the findings, timeliness improved for BCG and Penta 3 but remained slightly unchanged for Penta 1 and 2, most likely because BCG is an initiation vaccine into the routine vaccination schedule and mothers are keen for their babies to get vaccinated. For Penta 1 and Penta 2, mothers are not very keen on the time interval between the two vaccines as long as their babies get vaccinated. For Penta 3 , mothers are always keen on completing the vaccine series hence the drive to bring their babies to the clinic. Another study conducted in Vietnam to establish the impact of mhealth on improving immunization coverage among children reported an increase in timeliness for vaccination with Polio 1, Measles, and Quinvaxem, one-year post-intervention [16]. This is consistent with our study findings.

Since most documented studies are on electronic vaccine registries that are web-based, more work needs to be done to explore the contribution of USSD in the creation of electronic vaccine registries.

\section{Conclusion}

We successfully developed a platform that can register births, record vaccines, and send automated SMS reminders using USSD technology. As the system uses low-cost phones, we saved more than $60 \%$ on the cost of phones and about $30-40 \%$ on the system monthly charges compared if we had used smartphones that require data bundles. Therefore, we believe that the system has great potential to improve timeliness and vaccination coverage, especially if applied in hard-to-reach areas and urban populations where most vaccination defaulters are found. We recommend that electronic vaccination registries should use a combination of both USSD and internet-based technology, so us to serve users from all settings.

\section{Acknowledgements}

We acknowledge the support given by the health workers and field team that piloted the system. Special thanks go to Bill and Melinda Gates Foundation for financing the project.

\section{Conflict of Interest}

The authors declare no conflict of interest. 


\section{References}

[1] W. Orenstein and R. Ahmed, "Simply put: Vaccination saves lives," 2017.

[2] M. Songane, "Challenges for nationwide vaccine delivery in African countries," Inter. J. Heal. Econ. Manag. 2017 182, vol. 18, no. 2, pp. 197-219, Oct. 2017.

[3] M. L. Mallory, L. C. Lindesmith, and R. S. Baric, "Vaccination-induced herd immunity: Successes and challenges," J. Allergy Clin. Immunol., vol. 142, no. 1, pp. 64-66, Jul. 2018.

[4] N. C. Galles et al., "Measuring routine childhood vaccination coverage in 204 countries and territories, 1980-2019: a systematic analysis for the Global Burden of Disease Study 2020, Release 1," Lancet, vol. 398, no. 10299, pp. 503-521, Aug. 2021.

[5] M. C. Danovaro-Holliday, E. Dansereau, D. A. Rhoda, D. W. Brown, F. T. Cutts, and M. GacicDobo, "Collecting and using reliable vaccination coverage survey estimates: Summary and recommendations from the 'Meeting to share lessons learnt from the roll-out of the updated WHO Vaccination Coverage Cluster Survey Reference Manual and to set an operational research agenda around vaccination coverage surveys', Geneva, 18 21 April 2017," Vaccine, vol. 36, no. 34, pp. 51505159, Aug. 2018.

[6] J. J. Rainey, M. Watkins, T. K. Ryman, P. Sandhu, A. Bo, and K. Banerjee, "Reasons related to non-vaccination and under-vaccination of children in low and middle-income countries: Findings from A systematic review of the published literature, 1999-2009," Vaccine, vol. 29, no. 46, pp. 82158221, Oct. 2011.

[7] H. J. Larson, C. Jarrett, E. Eckersberger, D. M. D. Smith, and P. Paterson, "Understanding vaccine hesitancy around vaccines and vaccination from a global perspective: A systematic review of published literature, 2007-2012," Vaccine, vol. 32, no. 19, pp. 2150-2159, Apr. 2014.

[8] M. de C. Tauil, A. P. S. Sato, and E. A. Waldman, "Factors associated with incomplete or delayed vaccination across countries: A systematic review," Vaccine, vol. 34, no. 24, pp. 2635-2643, May 2016.
[9] M. Favin, R. Steinglass, R. Fields, K. Banerjee, and M. Sawhney, "Why children are not vaccinated: a review of the grey literature," Int. Health, vol. 4, no. 4, pp. 229-238, Dec. 2012.

[10]C. S. Wiysonge, O. A. Uthman, P. M. Ndumbe, and G. D. Hussey, "Individual and Contextual Factors Associated with Low Childhood Immunisation Coverage in Sub-Saharan Africa: A Multilevel Analysis," PLoS One, vol. 7, no. 5, p. e37905, May 2012.

[11]A. T. Kroger, W. L. Atkinson, E. K. Marcuse, and L. K. Pickering, "General recommendations on immunization; recommendations of the Advisory Committee on Immunization Practices (ACIP)," MMWR. Recommendations and reports : Morbidity and mortality weekly report. Recommendations and reports / Centers for Disease Control, vol. 55, no. RR-15. pp. 1-48, 2006.

[12] J. C. Jacobson Vann and P. Szilagyi, "Patient reminder and recall systems to improve immunization rates," Cochrane Database Syst. Rev., vol. 2017, no. 10, Jul. 2005.

[13] "Demographic: Health Survey (2008). Dhs program. com/pubs/p - Google Scholar." [Online]. Available on

https://scholar.google.com/scholar?cluster $=7811762$ $480719835407 \& h l=$ en\&as_sdt $=2005 \&$ sciodt $=0,5$

[Accessed: 19-Jul-2021].

[14]"SciELO - Public Health - Electronic immunization registries in Latin America: progress and lessons learned Electronic immunization registries in Latin America: progress and lessons learned." [Online] Available on https://www.scielosp.org/article/rpsp/2014.v35n56/453-457/en/ [Accessed: 02-Oct-2021]. [15]A. E. Tozzi, F. Gesualdo, A. D’Ambrosio, E. Pandolfi, E. Agricola, and P. Lopalco, "Can Digital Tools Be Used for Improving Immunization Programs?," Front. Public Health., vol. 0, p. 36, Mar. 2016.

[16]N. T. Nguyen, H. M. Vu, S. D. Dao, H. T. Tran, and T. X. C. Nguyen, "Digital immunization registry: evidence for the impact of Health on enhancing the immunization system and improving immunization coverage for children under one year 
old in Vietnam," mHealth, vol. 3, pp. 26-26, Jul. 2017.

[17]M. S. Stockwell and A. G. Fiks, "Utilizing health information technology to improve vaccine communication and coverage," http://dx.doi.org/10.4161/hv.25031, vol. 9, no. 8, pp. 1802-1811, Aug. 2013.

[18]A. Odone et al., "Effectiveness of interventions that apply new media to improve vaccine uptake and vaccine coverage," https://doi.org/10.4161/hv.34313 vol. 11, no. 1, pp. 72-82, Jan. 2015.

[19] S. B. Dolan et al., "Redefining vaccination coverage and timeliness measures using electronic immunization registry data in low and middle income countries," Vaccine, vol. 37, no. 13, pp. 1859-1867, Mar. 2019. 